Lombardi V., Bombaci A. et al,

Plasma pNfH differentiate SBMA from ALS

\title{
Plasma pNfH differentiate SBMA from ALS
}

Vittoria Lombardi $\mathrm{PhD}^{* 1,2}$, Bombaci Alessandro $\mathrm{MD}^{* 2,3}$, Luca Zampedri ${ }^{1}$, ChingHua Lu MD, $\mathrm{PhD}^{1,4}$, Bilal Malik $\mathrm{PhD}^{5}$, Henrik Zetterberg $\mathrm{PhD}^{1,7,8,9}$, Amanda Heslegrave $\mathrm{PhD}^{1,7}$, Carlo Rinaldi $\mathrm{MD}, \mathrm{PhD}^{6}$, Linda Greensmith $\mathrm{PhD}^{1}$, Michael Hanna FRCP ${ }^{1}$, Andrea Malaspina MD, PhD, FRCP ${ }^{2}$ and Pietro Fratta MD, $\mathrm{PhD}^{1}$

${ }^{1}$ Institute of Neurology, University College London Institute of Neurology, Queen Square, London, WC1N 3BG, UK. ${ }^{2}$ Blizard Institute, Queen Mary, University of London, UK. ${ }^{3}$ "Rita Levi Montalcini" Department of Neuroscience, University of Torino, Turin, Italy. ${ }^{4}$ Department of Neurology, China Medical University Hospital, Taiwan. ${ }^{5}$ Basildon Hospital, Basildon, UK. ${ }^{6}$ Department of Paediatrics, University of Oxford, Oxford, OX1 3QX, UK. ${ }^{7}$ UK Dementia Research Institute at UCL, London WC1E 6BT, UK. ${ }^{8}$ Clinical Neurochemistry Laboratory, Sahlgrenska University Hospital, S-431 80 Mölndal, Sweden. ${ }^{9}$ Department of Psychiatry and Neurochemistry, Institute of Neuroscience and Physiology, the Sahlgrenska Academy at the University of Gothenburg, S-431 80 Mölndal, Sweden.

* These authors contributed equally to this work

\section{Address for correspondence:}

Dr Pietro Fratta

University College London Institute of Neurology,

Queen Square, London, WC1N 3BG, UK.

Tel: +44 (0)2034483899 Fax: +44 (0)20 43383111

Email:p.fratta@ucl.ac.uk

and

Dr Andrea Malaspina

Queen Mary University

4 Newark St, London, E1 2AT, UK.

Email: a.malaspina@qmul.ac.uk

Title Character count: 6

Text word count: 1500

Abstract words count: 198

Figures: 1

Tables: 1

References: 15

\section{Financial Disclosures:}

All authors report no disclosures

Study Funded by the NIHR UCLH Biomedical Research Centre Grant \#BRC279566 


\section{Abstract}

Background and aim: Spinal bulbar muscular atrophy (SBMA) is a progressive adult-onset X-linked neuromuscular disease. Although traditionally considered a motor neuron disorder, recent advances have highlighted a primary myopathic component. We evaluated levels of phosphorylated neurofilament heavy chain (pNfH), a known biomarker for neurodegeneration, in SBMA.

Materials and methods: We collected plasma and serum from 46 SBMA, 50 ALS and 50 healthy control cases, alongside with plasma from a mouse model of SBMA (AR100) and littermate controls. We measured pNfH plasma levels using Single molecule array (Simoa), we assessed functional scales and we gathered demographic data. We analysed data using Mann-Whitney $\mathrm{U}$ test, Kruskal-Wallis test and Cox regression analysis.

Results: Plasma pNfH levels were significantly increased in ALS, but, intriguingly, there was no change in SBMA. These results were also confirmed in SBMA mice. The ROC curve highlighted that $\mathrm{pNfH}$ levels can effectively distinguish between ALS and SBMA (AUC 0.95).

Conclusions: Unexpectedly, levels of $\mathrm{pNfH}$ are normal in SBMA, whilst they are increased in ALS, and suggest pNfH could serve as a biomarker to differentiate the two diseases. Further, this finding is in agreement with recent evidence showing that primary muscle damage is a crucial feature in SBMA.

Keywords: Kennedy Disease, Spinal Bulbar Muscle Atrophy, Heavy chain Neurofilaments, Biomarker, Amyotrophic Lateral Sclerosis 
Lombardi V., Bombaci A. et al, Plasma pNfH differentiate SBMA from ALS

\section{Introduction}

Spinal and bulbar muscular atrophy (SBMA), also known as Kennedy disease $(\mathrm{KD})$, is a slowly progressive adult-onset X-linked neuromuscular disorder any effective treatments. It is characterized by limb and bulbar muscle wasting, weakness and fasciculation, associated with metabolic and endocrine alterations ${ }^{1,2}$. SBMA is caused by the expansion of a CAG repeat in exon 1 of the androgen receptor $(A R)$ gene; more than 37 repeats are pathogenic ${ }^{1}$.

There is a strong need for disease activity and progression markers, which are necessary for effective clinical trials. Furthermore, although the genetic test is diagnostic, there is a lack of biomarkers to aid the initial differential diagnosis of the disease. Neurofilaments (Nfs), both light and heavy chains, are now becoming a widely accepted prognostic biomarker for amyotrophic lateral sclerosis (ALS) and other neurodegenerative diseases ${ }^{3}$. More than twenty years ago neurofilaments were first found to be increased in $\mathrm{ALS} \mathrm{CSF}^{4}$. Reliable measurements of Nfs in peripheral biofluids have become available over the last five years, NfL were found to predict disease course in ALS and both NfL and $\mathrm{pNfH}$ are increased in a wide range of neurological conditions ${ }^{3,5,6}$. Nfs are currently being used as a biomarker in numerous clinical trials.

Recently, plasma NfL levels were found to be normal in SBMA patients ${ }^{14}$. This finding has supported other lines of evidence that point to a primary myopathic component in SBMA ${ }^{2,7,8}$.

We here investigated pNfH plasma levels in SBMA patients and in a wellestablished rodent model of disease, using the highly sensitive Simoa platform.

\section{Materials and Methods}

\section{Study design}

This is a cross-sectional study in which we enrolled SBMA and ALS patients, visited in Motor neuron clinics at University College of London Hospital and at Queen Mary Hospital between Sep 2009 and Nov 2017. We included 46 SBMA patients, 50 ALS patients and 50 healthy controls (HCs). ALS were subdivided in 2 sub-groups (ALS-Fast and ALS-Slow) on the basis of their disease progression rate to last visit (PRL): PRL ALS-Slow<0.6 and ALS-Fast >0.9. PRL was calculated as 48 (corresponding to the in-healthy state, before symptoms onset) 
minus the ALS Functional-Rating-Scale revised score (ALSFRSr) at the last visit, divided by time interval in months between symptoms onset and last visit date.

We collected plasma after patients' written informed consent. Approvals were obtained from the East London and the City Research Ethics Committee (09/H0703/27).

We also assessed the test in mice, 10 well-established mouse models of SBMA (AR100) and 10 wild type littermate controls.

Samples were processed, stored and analysed as previously described ${ }^{5}$. All blood samples were collected into EDTA-containing tubes, centrifuged at $20^{\circ} \mathrm{C}$ at 3.500 rpm for 10 minutes within 1 hour and stored at $-80^{\circ} \mathrm{C}$.

We measured pNfH levels in plasma performing a single molecule array (Simoa)based assay (Quanterix, Lexington, MA) ${ }^{9}$, using pNF-Heavy Discovery Kit 102669.

\section{Clinical assessment}

SBMA patients had a genetical confirm of diagnosis, while ALS patients had a diagnosis of definite or probable ALS according to the revised El-Escorial criteria $^{10}$. Disease severity was assessed using ALSFRSr ${ }^{11}$, SBMA FunctionalRating-Scale (SBMAFRS) ${ }^{12}$ and Adult-Myositis-Assessment-Tool (AMAT) ${ }^{13}$ scale in SBMA patients and using ALSFRS-r scale in ALS patients. Demographic and clinical data of patients are gathered in Table 1.

\section{Statistical analysis}

Mann-Whitney $U$ test and Kruskal-Wallis tests were performed to analyse plasma pNfH levels between groups. Dunn's multiple comparisons test was performed following Kruskal-Wallis test in case of significant differences. Receiver operating characteristic (ROC) curve, and the corresponding sensitivity, specificity, positive and negative predictive values, accompanied by their $95 \% \mathrm{Cls}$, was performed in order to identify the best cut-off level of pNfH to separate ALS and SBMA patients. Correlation between parameters was calculated by Spearman rank correlation $r$. The level of significance for all statistical tests was set at 0.05 . The program Prism V.8 (GraphPad Software, La Jolla, CA) was used to perform statistical calculations. 
Lombardi V., Bombaci A. et al, Plasma pNfH differentiate SBMA from ALS

\section{Results}

This study included 46 patients with SBMA, 50 ALS patients and 50 HCs. Participant demographic and clinical characteristics are summarized in Table 1.

We measured $\mathrm{pNfH}$ on plasma samples using a Simoa assay and found levels to be unchanged in SBMA vs HCs (Figure 1A). Of note, mean pNfH levels in SMBA were lower than HCs, although this difference was not statistically significant using Dunn's multiple comparison test. These results were also confirmed in SBMA mice, where $\mathrm{pNfH}$ levels were lower than WT mice $(p=0,009)$ (Figure 1B). Conversely, in both fast- and slow-progressing ALS groups there was a statistically significant increase of plasma pNfH levels compared to HCs (ALSSlow $p<0.001$; ALS-Fast $p<0.0001$ ), conforming with previous reports ${ }^{3,5}$. The two groups significantly differed when performing a Mann-Whitney test $(p=0.012)$, although significance was not retained after Dunn's multiple comparison correction. Therefore, in order to further investigate whether $\mathrm{pNfH}$ levels are associated to disease severity in ALS, we tested the correlation between $\mathrm{pNfH}$ levels and disease progression rate and observed a moderately significant association $\left(r_{\mathrm{s}}=0.36, \mathrm{p}=0.01\right)$.

We then tested whether $\mathrm{pNfH}$ levels correlate with disease functional impairment. No correlation was found between $\mathrm{pNfH}$ and ALSFRSr, SBMAFRS and AMAT scales in SMBA patients $\left(r_{s}=-0.029, p=0.86 ; r_{s}=0.025, p=0.89 ; r_{s}=0.024, p=0.89\right.$, respectively) or between $\mathrm{pNfH}$ and ALSFRSr in ALS patients $\left(r_{s}=-0.193, p=0.18\right)$. pNfH did not correlate with age in all groups.

Importantly, pNfH plasma levels were significantly different between SBMA and both ALS groups $(\mathrm{p}<0.0001)$. In order to identify the $\mathrm{pNfH}$ threshold to most effectively distinguish SBMA from ALS, we created a ROC curve (AUC=0.95, $\mathrm{p}<0.0001$; Figure 1D) and defined $\mathrm{pNfH}$ of $105 \mathrm{pg} / \mathrm{ml}$ (highest Youden-Index) as the best cut-off between ALS and SBMA (98\% sensitivity, 86\% specificity). Given the similarity of results previously reported with $\mathrm{NfL}$, we compared $\mathrm{pNfH}$ with previously measured $\mathrm{NfL}$ levels, in the same samples ${ }^{14}$. Cox regression model analysis, showed a significant correlation between $\mathrm{pNfH}$ and $\mathrm{NfL}$ levels $\left(r_{\mathrm{s}}=0.77\right.$, $\mathrm{p}<0.0001$; Figure 1C). 


\section{Discussion}

Contrary to what would be expected in a motor cell disorder ${ }^{3,14,15}$, this study shows low levels of $\mathrm{pNfH}$ in SBMA patients rather than a disease-related increase, as demonstrated in ALS and in primary lateral sclerosis. The result was also confirmed in a well-established SBMA mouse model.

We highlighted an increase of pNfH levels in ALS, and, beyond to what previously reported $^{15}$, we have also found a correlation between $\mathrm{pNfH}$ levels and disease progression rate in ALS (calculated as PRL). As pNfH levels are increased in the initial phases of $A L S^{15}$, this finding could be complementary to genetics to provide an early differential diagnosis for SBMA and other lower motor neuron diseases. It could also bear importance in predicting disease progression and in patient stratification for clinical trials.

We have also seen no relationships between $\mathrm{pNfH}$ levels and the stage of disease (quantified using functional and motor scales) both in SBMA and in ALS patients. This could suggest an absence of variation of $\mathrm{pNfH}$ levels during the course of these diseases, confirming previous data obtained in ALS with in-house ELISA test ${ }^{15}$.

Moreover, $\mathrm{pNfH}$ and $\mathrm{NfL}$ measurements in the same patients showed a good correlation; this correlation is tighter in ALS group than SBMA and HCs groups, due to the highest variability of measurement when detecting low levels of neurofilaments. Further work will be needed to assess whether combining these measurements could ameliorate the predictive value of the test.

Using ROC curve, we were also able to identify a statistically strong cut-off level $(A U C=0.95)$ of $\mathrm{pNfH}$ that could distinguish SBMA from ALS. This suggests that $\mathrm{pNfH}$ could be used as a biomarker for the differential diagnosis between SBMA and ALS.

Limits of this study are represented by the cross-sectional design that does not allow us to infer about the variation of $\mathrm{pNfH}$ during the disease course. With regard to the analytical aspect, in this study we employed one of the most sensitive platforms for neurofilament analysis; this approach was the same used 
for the NfL study ${ }^{14}$. Unlike the measurement of $\mathrm{NfL}$, previous work on the detection of plasma neurofilament isoforms has highlighted the inherent difficulties encountered in pNfH measuring in biological fluids, including the lack of linearity in dilution experiments and the presence of $\mathrm{pNfH}$ immunocomplexes or aggregates in circulation; that could cause epitope masking and make measurements for clinical stratification difficult ${ }^{15}$.

Finally, normal levels of pNfH in SBMA are in contrast not only with ALS, but also with previous findings on peripheral neuropathies ${ }^{3,5}$, that share a slow disease course, although have a mild to moderate $\mathrm{pNfH}$ increase. Unfortunately, the latter data resulted from small cohorts of patients, included in largest studies, or from case reports and pNfH was measured using in-house ELISA test; so there could have been an underestimation of results.

This data supports the recent hypothesis of a primary muscle origin of damage in SBMA patients ${ }^{2,4,13,14}$ and is consistent with results of NfL in SBMA ${ }^{14}$.

Under this light, it could be useful to measure, in a prospective study, pNfH levels in primary muscle atrophy and other pure lower motor neuron diseases, using Simoa platform.

\section{Conclusions}

In conclusion, we have demonstrated that plasma pNfH concentrations are not increased in SBMA patients and in a mouse model of disease, as opposed to ALS, and that $\mathrm{pNfH}$ could be used as a biomarker in differential diagnosis between SBMA and ALS.

\section{Acknowledgements}

We thank the patients involved in the study and their families for the participation and support to KD research. All samples were obtained from the ALS Biomarker study. PF is supported by an MRC/MNDA LEW Clinician Scientist Fellowship and the NIHR UCLH BRC. This study was supported by the NIHR UCLH BRC, the UCL Kennedy's Disease Fund and KDUK. OJZ is funded by the UK National Institute of Health Research on an Academic Clinical Fellowship. CR is funded by a Welcome Trust Clinical Research Career Development Fellowship and the Muscular Dystrophy Association (MDA). HZ is funded by the UK Dementia 
bioRxiv preprint doi: https://doi.org/10.1101/549576; this version posted February 14,2019 . The copyright holder for this preprint (which was not certified by peer review) is the author/funder, who has granted bioRxiv a license to display the preprint in perpetuity. It is made available under aCC-BY-NC-ND 4.0 International license.

Lombardi V., Bombaci A. et al, Plasma pNfH differentiate SBMA from ALS

Research Institute at UCL, the European Research Council, the Swedish Research Council and the Knut and Alice Wallenberg Foundation. The Simoa instrument was bought using a Welcome Trust multi-user equipment grant (PI: $H Z)$.

\section{References:}

1. Querin G, Sorarù G, Pradat PF. Kennedy disease (X-linked recessive bulbospinal neuronopathy): A comprehensive review from pathophysiology to therapy. Rev Neurol (Paris). 2017;173(5):326-337. doi:10.1016/j.neurol.2017.03.019

2. La Spada A. Spinal and Bulbar Muscular Atrophy. University of Washington, Seattle; 1993. http://www.ncbi.nlm.nih.gov/pubmed/20301508. Accessed February 7, 2019.

3. Steinacker P, Feneberg E, Weishaupt J, et al. Neurofilaments in the diagnosis of motoneuron diseases: A prospective study on 455 patients. J Neurol Neurosurg Psychiatry. 2016;87(1):12-20. doi:10.1136/jnnp-2015-311387

4. Rosengren LE, Karlsson J-E, Karlsson J-O, Persson LI, Wikkelsø C. Patients with Amyotrophic Lateral Sclerosis and Other Neurodegenerative Diseases Have Increased Levels of Neurofilament Protein in CSF. J Neurochem. 2002;67(5):2013-2018. doi:10.1046/j.1471-4159.1996.67052013.x

5. Feneberg E, Oeckl P, Steinacker $P$, et al. Multicenter evaluation of neurofilaments in early symptom onset amyotrophic lateral sclerosis. Neurology. 2018;90(1):e22-e30. doi:10.1212/WNL.0000000000004761

6. C.-H. L, C. M-W, E. G, et al. Neurofilament light chain: A prognostic biomarker in amyotrophic lateral sclerosis. Neurology. 2015;84(22):2247-2257. doi:10.1212/WNL.0000000000001642

7. Manzano R, Sorarú G, Grunseich C, et al. Beyond motor neurons: expanding the clinical spectrum in Kennedy's disease. J Neurol Neurosurg Psychiatry. 2018;89(8):808-812. doi:10.1136/jnnp-2017-316961

8. Dahlqvist JR, Oestergaard ST, Poulsen NS, Thomsen C, Vissing J. Refining the spinobulbar muscular atrophy phenotype by quantitative MRI and clinical assessments. Neurology. 2019;92(6):e548-e559. doi:10.1212/WNL.0000000000006887

9. Rissin DM, Kan CW, Campbell TG, et al. Single-molecule enzyme-linked immunosorbent assay detects serum proteins at subfemtomolar concentrations. Nat Biotechnol. 2010;28(6):595-599. doi:10.1038/nbt.1641

10. Brooks BR, Miller RG, Swash M, Munsat TL, World Federation of Neurology Research Group on Motor Neuron Diseases. El Escorial revisited: revised criteria for the diagnosis of amyotrophic lateral sclerosis. Amyotroph Lateral Scler Other Motor Neuron Disord. 2000;1(5):293-299. http://www.ncbi.nlm.nih.gov/pubmed/11464847. Accessed February 7, 2019.

11. Cedarbaum JM, Stambler N, Malta E, et al. The ALSFRS-R: a revised ALS functional rating scale that incorporates assessments of respiratory function. BDNF ALS Study Group (Phase III). J Neurol Sci. http://www.ncbi.nlm.nih.gov/pubmed/10540002. Accessed February 7, 2019.

12. Hashizume A, Katsuno M, Suzuki K, et al. A functional scale for spinal and bulbar muscular atrophy: Cross-sectional and longitudinal study. Neuromuscul Disord. 2015;25(7):554-562. doi:10.1016/j.nmd.2015.03.008

13. Harris-Love MO, Fernandez-Rhodes L, Joe G, et al. Assessing function and endurance in adults with spinal and bulbar muscular atrophy: validity of the adult myopathy assessment tool. Rehabil Res Pract. 2014;2014:873872. doi:10.1155/2014/873872

14. Lombardi V, Querin G, Ziff OJ et al. Neuron and muscle biomarkers for SBMA. Neurology 2019;92:1-7. doi:10.1212/WNL.0000000000007097

15. Lu C-H, Petzold A, Topping J, et al. Plasma neurofilament heavy chain levels and disease progression in amyotrophic lateral sclerosis: insights from a longitudinal study. J Neurol Neurosurg Psychiatry. 2015;86(5):565-573. doi:10.1136/jnnp-2014-307672 
Table 1 - Cohort demographic and genetic information, pNfH and scales values

\begin{tabular}{|c|c|c|c|c|c|c|c|c|c|}
\hline $\begin{array}{l}\text { Patients } \\
\text { Group }\end{array}$ & $n^{\circ}$ & $\begin{array}{l}\text { Sex } \\
M / F\end{array}$ & $\begin{array}{c}\text { Age } \pm \\
\text { SD } \\
(y)\end{array}$ & $\begin{array}{c}\mathbf{n}^{\circ} \\
\mathrm{CAG} \pm \\
\mathrm{SD}\end{array}$ & $\begin{array}{l}\text { PRL } \pm \\
\text { SEM }\end{array}$ & $\begin{array}{c}\text { pNfH } \pm \\
\text { SEM } \\
(p g / m l)\end{array}$ & $\begin{array}{l}\text { ALSFRSr } \\
\pm \text { SEM }\end{array}$ & $\begin{array}{c}\text { SBMAFRS } \\
\pm \text { SEM }\end{array}$ & $\begin{array}{c}\text { AMAT } \pm \\
\text { SEM }\end{array}$ \\
\hline SBMA & 46 & $46 / 0$ & $\begin{array}{c}56,6 \pm \\
11,1\end{array}$ & $\begin{array}{c}42.1 \pm \\
0.4\end{array}$ & $\mathrm{~N} / \mathrm{A}$ & $42 \pm 4.9$ & $\begin{array}{c}38.8 \pm 0.8 \\
(n=37)\end{array}$ & $\begin{array}{c}41.1 \pm 1.3 \\
(n=36)\end{array}$ & $\begin{array}{c}31.8 \pm \\
1.4 \\
(n=38)\end{array}$ \\
\hline $\begin{array}{l}\text { ALS- } \\
\text { Slow }\end{array}$ & 25 & $14 / 11$ & $\begin{array}{c}66,9 \pm \\
12,7\end{array}$ & $\mathrm{~N} / \mathrm{A}$ & $\begin{array}{c}0.27 \pm \\
0.04\end{array}$ & $\begin{array}{c}629 \pm \\
159\end{array}$ & $37.6 \pm 1.6$ & $\mathrm{~N} / \mathrm{A}$ & $\mathrm{N} / \mathrm{A}$ \\
\hline $\begin{array}{l}\text { ALS- } \\
\text { Fast }\end{array}$ & 25 & $10 / 15$ & $\begin{array}{c}66.4 \pm \\
10.7\end{array}$ & $\mathrm{~N} / \mathrm{A}$ & $\begin{array}{l}1.61 \pm \\
0.12\end{array}$ & $\begin{array}{c}1615 \pm \\
327\end{array}$ & $31.7 \pm 1.8$ & $\mathrm{~N} / \mathrm{A}$ & $\mathrm{N} / \mathrm{A}$ \\
\hline $\mathrm{HC}$ & 50 & $17 / 33$ & $\begin{array}{l}58,5 \\
(6,8) \\
\end{array}$ & $\mathrm{N} / \mathrm{A}$ & $\mathrm{N} / \mathrm{A}$ & $\begin{array}{l}76 \pm \\
12.7 \\
\end{array}$ & N/A & $\mathrm{N} / \mathrm{A}$ & $\mathrm{N} / \mathrm{A}$ \\
\hline
\end{tabular}

Abbreviations: Healthy controls (HCS), spinal bulbar muscular atrophy (SBMA); fast progressing amyotrophic lateral sclerosis (ALS-Fast); slow progressing amyotrophic lateral sclerosis (ALS-Slow); standard deviation (SD), standard error of the mean (SEM); years $(\mathrm{y})$; female $(\mathrm{F})$; male $(\mathrm{M})$; number $\left(\mathrm{n}^{\circ}\right)$; number of CAG repeats in androgen receptor gene ( $\left.n^{\circ} \mathrm{CAG}\right)$; adult myopathy assessment tool (AMAT) ; spinal bulbar muscular atrophy function rating scale (SBMAFRS); amyotrophic lateral sclerosis function rating revised scale (ALSFRSr); phosphorylated neurofilament heavy chain $(\mathrm{pNfH})$; progression rate to last visit (PRL). 

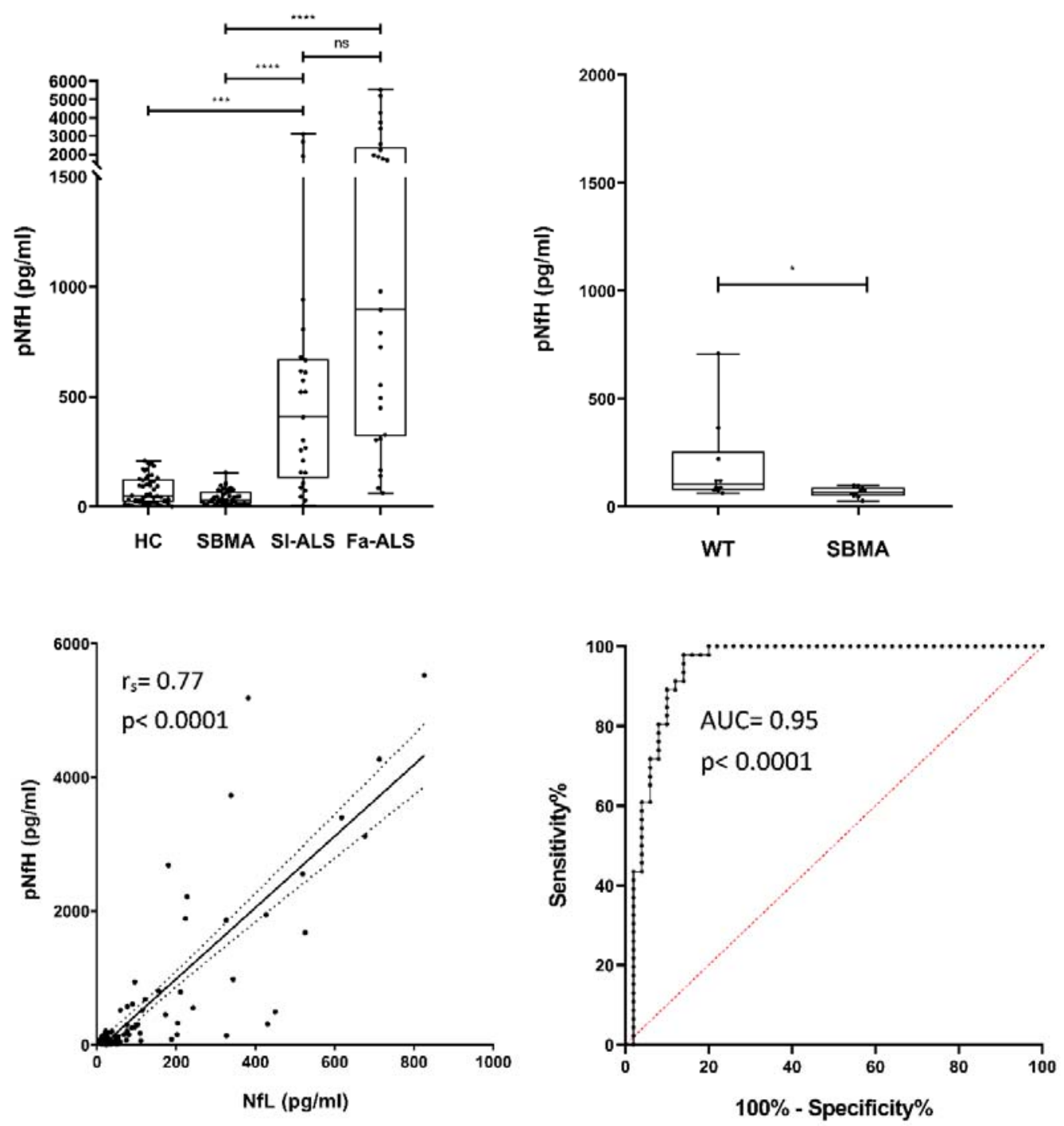

\section{Finure 1}

\section{A}

$\left(t, \ldots . \mathrm{JfH}\right.$ concentration in HCs, SBMA, $f^{B}$ Slow and ALS-Fast. Using Kruskal-Wallis test and Dunn's multiple comparison test we observed reduced levels of both $\mathrm{pNfH}$ compared to ALS groups (all $p$-value < 0.0001). (B) $\mathrm{pNfH}$ levels from mice AR100 (SBMA) and littermate controls (WT) show a light statistically significant difference (Mann Whitney test). (C) Correlation between $\mathrm{pNfH}$ and $\mathrm{NfL}\left(r_{\mathrm{s}}=0.77, \mathrm{p}<0.0001\right)$. (D) ROC curve, created comparing pNfH and NfL levels, shows a high AUC $(0.95 ; 95 \% \mathrm{Cl} 0.90-$ $1.00)$. The value associated to the highest Youden-Index is $105 \mathrm{pg} / \mathrm{ml}(98 \%$ sensitivity, $86 \%$ specificity, PPV 0.88, NPV 0.98).

Fa-ALS= Fast-progressing amyotrophic lateral sclerosis; SI-ALS= slow-progressing amyotrophic lateral sclerosis; $\mathrm{WT}=$ wild type mice; $\mathrm{HCs}=$ Healthy controls; $\mathrm{SBMA}=$ spinal bulbar muscular atrophy; $\mathrm{pNfH}=$ phosphorylated-Heavy-Neurofilaments; $\mathrm{NfL}=$ Light-Chain-Neurofilaments; $\mathrm{AUC}=$ area under curve; $P P V=$ positive predictive value: $N P V=$ negative predictive value; $p=p$-value.

ns $=$ not statistically significant result; ${ }^{*} p<0.05 ;{ }^{* *} p<0.01 ;{ }^{* * *} p<0.001 ;{ }^{* * * *} p<0.0001$. 